\title{
Cross-Interface Emulsification for Generating Size-Tunable Droplets
}

Article in Analytical Chemistry · February 2016

DOI: 10.1021/acs.analchem.5b04510

CITATIONS

7

4 authors, including:

\section{Peng Xu}

University of California, San Francisco

2 PUBLICATIONS 8 CITATIONS

SEE PROFILE

Wenbin Du

Chinese Academy of Sciences

52 PUBLICATIONS 1,581 CITATIONS

SEE PROFILE

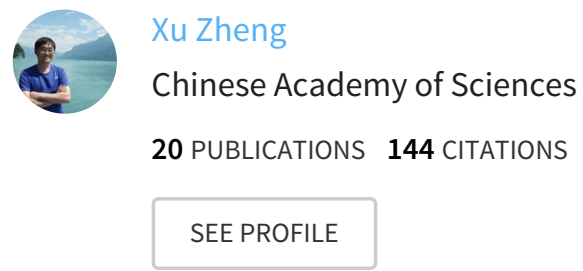

Some of the authors of this publication are also working on these related projects: 


\title{
Cross-Interface Emulsification for Generating Size-Tunable Droplets
}

\author{
$\underline{\text { Peng Xu}}{ }^{\dagger}$ Xu Zheng, ${ }^{\ddagger}$ Yi Tao, ${ }^{\dagger}$ and Wenbin $\underline{D u}^{* \dagger}$ \\ ${ }^{\dagger}$ State Key Laboratory of Microbial Resources, Institute of Microbiology, Chinese Academy of Sciences, Beijing 100101, China \\ ${ }^{\ddagger}$ State Key Laboratory of Nonlinear Mechanics, Institute of Mechanics, Chinese Academy of Sciences, Beijing 100190, China
}

Supporting Information

ABSTRACT: We report cross-interface emulsification (XiE), a simple method for the generation of monodisperse droplets of controllable volumes from picoliter to nanoliter. A device is set up in which a fused-silica capillary is vibrating across the surface of the continuous phase (mineral oil) in a reservoir, and the flow of the dispersed phase (aqueous solution) in the capillary is segmented into monodisperse droplets at the air/oil interface. We find that the volume of droplets is mainly dominated by the flow rate and vibrating frequency and not significantly influenced by other factors, such as the viscosity of the continuous phase and dispersed phase, the inner diameter of the capillary $(20-100 \mu \mathrm{m})$, or the shape of the tip (tapered or flat). These features reflect high robustness, flexibility, and precision of $\mathrm{XiE}$ for on-demand volume control of droplets. The droplets automatically assemble into planar monolayer droplet arrays (PMDA) in flat-bottomed microwells of 96-well plates, offering excellent convenience for imaging of droplets. As a representative application, we carry out
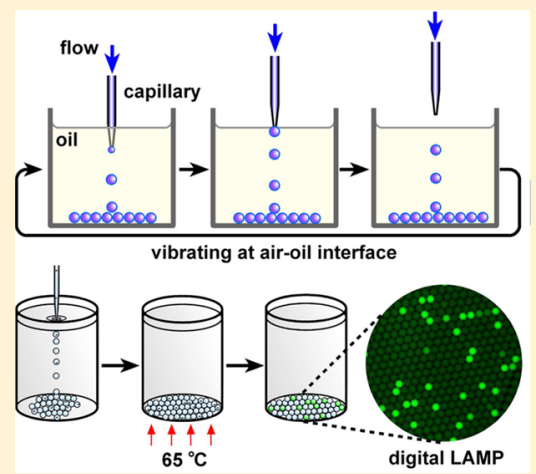
digital loop-mediated isothermal amplification using PMDAs with multivolume droplets for the absolute quantification of nucleic acids. Our results demonstrate that $\mathrm{XiE}$ is simple and controllable for the production of monodisperse size-tunable droplets, and it offers opportunities for common laboratories, even without microfabrication facilities, to perform digital quantification, single cell analysis, and other biochemical assays with high throughput.

$\mathrm{E}$ mulsions are mixtures of at least two immiscible fluids, one being dispersed in the other in the form of droplets, in the presence of surface active agents, typically surfactant molecules. ${ }^{1}$ They are ubiquitous in our daily life and have wide industrial applications, such as for foods, pharmaceuticals, cosmetics, and so forth. ${ }^{2}$ Traditionally, emulsions can be obtained via different kinds of energy input, such as shaking, ${ }^{3}$ stirring, ${ }^{4}$ homogenizing, ${ }^{5}$ or exposure to ultrasound. ${ }^{6}$ However, these methods produce droplets with a wide size distribution, which strongly influences the emulsion stability. ${ }^{7}$ Enormous efforts have been dedicated to producing monodisperse droplets using alternative techniques, such as membrane emulsification, ${ }^{8}$ spraying, and inkjet printing. ${ }^{10}$ Nevertheless, new approaches for monodisperse emulsification with precise size control are highly desirable.

The recent development of droplet-based microfluidics allows for the rapid generation and manipulation of monodisperse droplets in microfabricated devices. ${ }^{11}$ Three major geometries, including $\mathrm{T}$-junction, ${ }^{12}$ flow-focusing, ${ }^{13}$ and coflowing, ${ }^{14}$ are introduced for droplet generation. However, these methods require designing and fabricating microfluidic devices using specialized facilities and complex procedures, which are not widely available. The size and uniformity of droplets produced by these methods are governed by capillary instabilities of the confluence of immiscible liquid phases. 15,16 The size and uniformity of droplets are affected by many factors, such as the viscosity of the fluids, interfacial tension, modification of channel surfaces, precision of the microfabrication, and so forth. ${ }^{17}$ Therefore, it is always challenging to precisely and robustly control droplet generation on demand in a wide volume range.

In this paper, we developed a simple method for the high throughput generation of monodisperse droplets based on the vibration of a capillary with a continuous flow of the dispersed phase at the interface of the continuous phase, which we termed as cross-interface emulsification (XiE). The droplet volume conforms to the calculation of dividing the flow rate by the vibrating frequency and thus can be precisely controlled on demand. Droplets can automatically assemble into a planar monolayer droplet array (PMDA) in flat-bottomed reservoirs, offering exceptional convenience for microscopically imaging thousands of droplets simultaneously. The utility of $\mathrm{XiE}$ is demonstrated by digital loop-mediated isothermal amplification (dLAMP) for the absolute quantification of DNA templates using multivolume PMDAs.

\section{EXPERIMENTAL SECTION}

Preparation of Capillaries. Fused-silica capillaries were purchased from Polymicro Technologies (Phoenix, AZ, USA). Flat tip capillaries ( $40 \mu \mathrm{m}$ i.d., $127 \mu \mathrm{m}$ o.d.) were used directly by cutting into $3 \mathrm{~cm}$ long pieces using a capillary cutting stone (Hampton Research, Aliso Viejo, CA, USA). Tapered tips were made by heating the center of $6 \mathrm{~cm}$ long capillaries $(100 \mu \mathrm{m}$

Received: November 27, 2015

Accepted: February 5, 2016

Published: February 5, 2016 


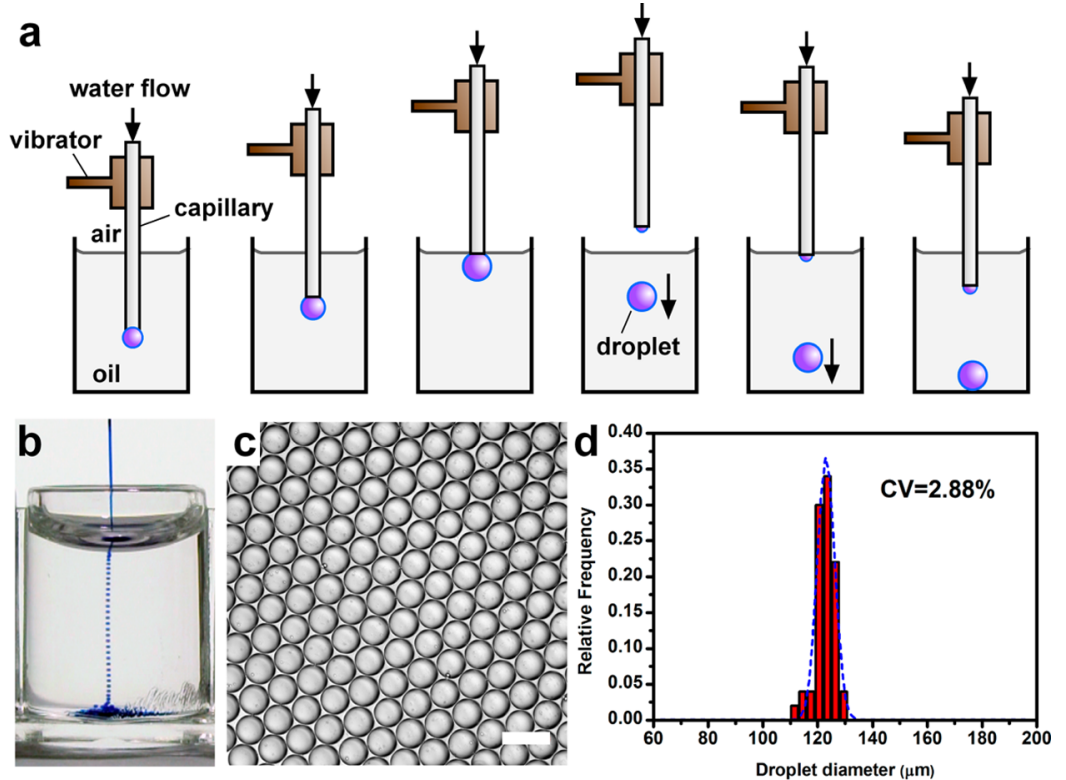

Figure 1. Droplet generation by cross-interface emulsification (XiE). (a) Schematic of a droplet generation cycle by XiE. (b) A picture of generating droplets of food dye solution in an oil-filled microwell. The capillary was vibrated at $50 \mathrm{~Hz}$ at the air/oil interface, as food dye solution was injected at a flow rate of $50 \mathrm{~nL} / \mathrm{s}$. (c) Monodisperse water droplets formed a planar monolayer droplet array (PMDA) at the bottom of the microwell. Scale bar $=200 \mu \mathrm{m}$. (d) The diameter distribution of droplets in (c).

i.d., $166 \mu \mathrm{m}$ o.d.) in a butane flame and simultaneously stretching the capillary from both ends manually; it was then cut in the middle into two pieces with fine tips. ${ }^{18}$ Tapered tips with 20,40 , and $60 \mu \mathrm{m}$ i.d. were selected after measuring using an inverted microscope (Eclipse Ti, Nikon Corp., Tokyo, Japan).

Setup of the XiE System. A simple system based on XiE was established (Figure S1). The capillary was fixed vertically by capillary wax (Hampton Research, Aliso Viejo, CA, USA) onto an electromagnetic vibrator with its amplitude and frequency controlled by a waveform generator (RIGOL Technologies, Beijing, China). The capillary was connected with the syringe pump via a $20 \mathrm{~cm}$ long Teflon tubing of 380 $\mu \mathrm{m}$ i.d. (Zeus, Orangeburg, SC, USA). The joint between the capillary and the Teflon tubing was sealed with capillary wax. The aqueous solution was loaded into a gastight syringe ( $50 \mu \mathrm{L}$ volume, Agilent, Santa Clara, CA, USA) and infused through the capillary by a syringe pump (Pump 11, PicoPlus Elite, Harvard Apparatus, Holliston, MA, USA). The microwells of 96-well plates (flat-bottom, Eppendorf, Hamburg, Germany) were filled with $300 \mu \mathrm{L}$ of oil. Unless otherwise indicated, the oil phase was a mixture of mineral oil (Sigma-Aldrich, St. Louis, MO, USA) and $n$-tetradecane (TCI, Beijing, China) at a ratio of 1:1 (v/v), containing surfactants including 3\% ABIL EM 90 (Evonik Industries, Essen, Germany). ${ }^{19}$ The viscosities of oils were measured by a viscometer (model NDJ-5S/8S, LICHEN group, Shanghai, China) at room temperature.

Droplet Generation by XiE. For generating droplets in the oil-filled microwells, the tip of the capillary was adjusted to approximately $1 \mathrm{~mm}$ above the oil surface and then started to vibrate at a constant frequency and peak amplitude $(\sim 2 \mathrm{~mm})$, which was controlled by a waveform generator. Next, the pump started infusion at a constant flow rate with user defined target volume. With a continuous flow of the aqueous solution in the capillary and its vibration across the oil surface, monodisperse droplets were obtained in the microwells (Figure 1b).
dLAMP. We first make the LAMP mix using solutions from the LoopAmp DNA amplification kit and the calcein fluorescence indicator kit (Eiken Chemical, Tokyo, Japan) according to the manufacturer's protocol in a PCR hood. A set of four primers designed by Primer Explorer were as follows: FIP: 5'-CAATGGAAAGCAGCAAATCCCGAGAGAATAGGGCGGTTA-3'; BIP: 5'-GCGTGTTTGTGCATCCATCTTCGTTCAGGACTACAACTG-3'; F3: 5'-TGATTTCGGTTTGCGATTC-3'; B3: 5'-GATTAGCTGCCAATGTGC-3'. They were synthesized by Sangon Biotechnology (Shanghai, China). The mix was supplemented with $1.0 \mathrm{mg} / \mathrm{mL}$ of bovine serum albumin (New England Biolabs, Ipswich, MA, USA). Stock lambda DNA (Takara, Dalian, China) solution with a starting concentration of $30 \mathrm{pg} / \mu \mathrm{L}$ was serial diluted with deionized water. Two microliters of the lambda DNA was used as the template, and the final volume of each reaction was 25 $\mu \mathrm{L}$. LAMP solutions were then loaded into the syringe to produce droplets using the $\mathrm{XiE}$ method using microwells of a 96-well plate as droplet reservoirs. The volume of droplets was adjusted by changing the flow rate. The plate was sealed with a film and incubated at $65{ }^{\circ} \mathrm{C}$ for $60 \mathrm{~min}$ to carry out isothermal DNA amplification.

Data Acquisition and Analysis. Images of droplet arrays were acquired using an Eclipse $\mathrm{Ti}$ microscope. The calcein fluorescence was imaged by a vacuum-cooled CMOS camera (model DC-152Q-C00-FI, Photometrics, Tucson, AZ, USA) through a GFP (exciter: $480 / 40 \mathrm{~nm}$; emitter: $527 / 30 \mathrm{~nm}$ ) filter cube. To cover a full PMDA in a microwell on the 96-well plate in a single micrograph, a $2 \times$ objective was used. Then, $10 \times$ and $20 \times$ objectives were used for imaging parts of the droplet array with higher resolution and sensitivity. All images were analyzed by ImageJ software (NIH, Bethesda, MD, USA).

\section{RESULTS AND DISCUSSION}

Origin and Validation of the XiE Idea. In nature, the female dragonfly lays eggs by rapidly tapping the surface of the water repeatedly with the tip of its abdomen. ${ }^{20}$ The eggs are 
trapped under the air/water interface when its abdomen is lifted out of the water. This inspired us to design the $\mathrm{XiE}$ method, which generates monodisperse droplets with the vibration of a capillary across the surface of the continuous phase, as the dispersed phase is flowing (Figure 1a). We set up a system as described in the Experimental Section (Figure S1), and tested the droplet generation using a food dye solution. At a flow rate of $50 \mathrm{~nL} / \mathrm{s}$ and $50 \mathrm{~Hz}$ vibration, we successfully generated monodisperse droplets by this $\mathrm{XiE}$ system. The lower-than-water density of the oil phase allowed for the settlement of droplets at the flat bottom of the microwell, automatically forming a PMDA (Figure $1 \mathrm{~b}$ and c, Movie S1). The average diameter of the droplets generated under this condition was $124 \mu \mathrm{m}$, and the coefficient of variation (CV) was $2.88 \%$ (Figure $1 \mathrm{~d}$ ), reflecting outstanding monodispersity of droplets generated by $\mathrm{XiE}$.

We next investigated forces involved in the droplet detachment at the air/oil interface, as shown in Figure 2 (see
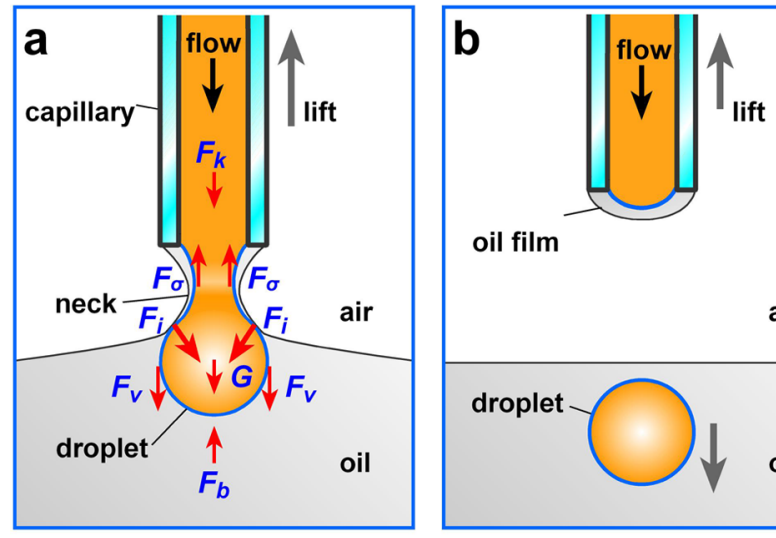

oil film
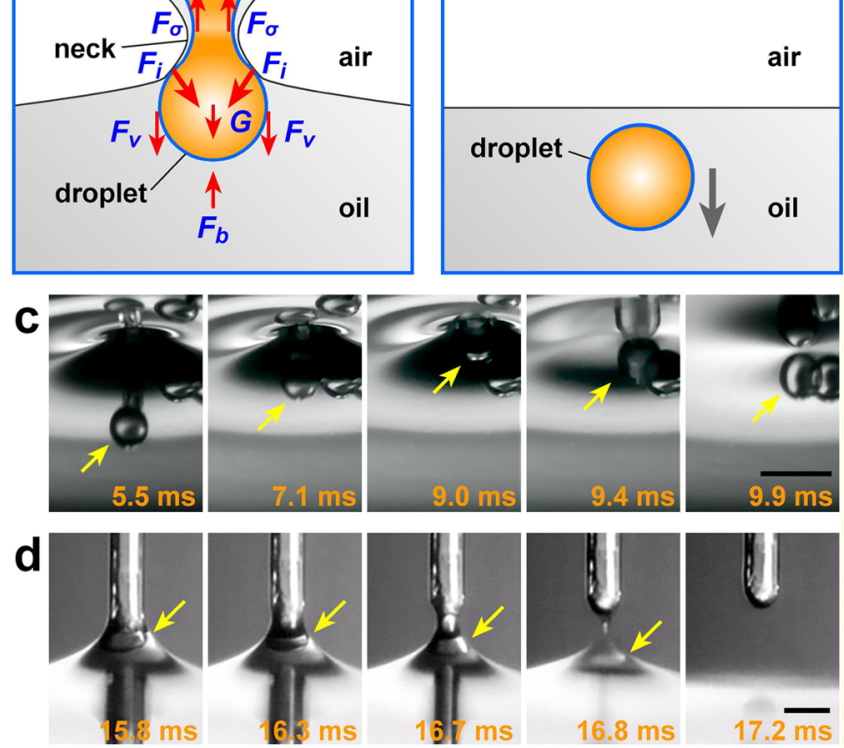

Figure 2. Principle and phenomenon of droplet generation by XiE. (a, b) Force analysis of the droplet detachment at the air/oil interface. Images taken by a high-speed video camera from under the oil (c) or above the oil (d) showing the detailed droplet generation process. Yellow arrows indicate a newly generated droplet. Scale bar $=200 \mu \mathrm{m}$.

Text S1 for details). We estimated the dimensionless parameters, such as capillary number $(\mathrm{Ca})$, Reynolds number $(\mathrm{Re})$, and the Bond number (Bo), ${ }^{21,22}$ and found that the surface tension effect was dominant whereas the inertial effect $\left(F_{\mathrm{k}}\right)$, gravity $(G)$, and the buoyant force $\left(F_{\mathrm{b}}\right)$ could be neglected. When the capillary tip was being lifted out of the carrier oil during vibration, the "blockage" due to the confinement of the air/oil interface $\left(F_{\mathrm{i}}\right)$ was found to be the key force in our $\mathrm{XiE}$ method that caused the droplet detachment by overcoming the connection in the neck (governed by water/oil interfacial force $F_{\sigma}$ ). Without $F_{\mathrm{j}}$, the viscous drag force $F_{\mathrm{v}}$ could hardly overcome $F_{\sigma}$ in the regime of small $\mathrm{Ca}$ (Figure 2a). A detailed discussion on the magnitude of the forces is given in Text S1. Our evaluation showed that the dominant force $F_{\mathrm{i}}$ was several orders larger than $F_{\sigma}$, which makes the droplet detachment easy and reproducible. After the droplet detached and the capillary left the oil, a residue layer of oil film sealed the outlet of the tip during its stay in the air, offering an effective contamination control of the aqueous solution (Figure $2 b$ and $d$ ). Details of the droplet generation process were recorded by a high-speed video camera, validating our hypothesis that the droplet detachment occurred at the air-oil interface and that the droplet generation frequency was the same as the vibrating frequency (Figure $2 \mathrm{c}$ and $\mathrm{d}$, Movies S2 and S3).

Droplet Size is Predictable and Controllable. From the high-speed video images, we could see that one droplet was generated per vibration cycle. Therefore, the volume of each droplet could be defined simply by

$$
V=\frac{Q}{f}
$$

where $Q$ is the flow rate $(\mathrm{nL} / \mathrm{s}), f$ is the vibrating frequency $\left(\mathrm{s}^{-1}\right)$, and $V$ is the droplet volume $(\mathrm{nL})$. To validate the effectiveness of eq 1 , we measured droplet sizes generated at different vibration frequencies $(25,50,100$, and $500 \mathrm{~Hz})$ and flow rates (50 and $75 \mathrm{~nL} / \mathrm{s})$. As shown in Figure 3, monodisperse droplets were formed under the tested conditions. When the flow rate was fixed, higher vibration frequencies generated smaller droplets; on the other hand, when the vibrating frequency was fixed, higher flow rates produced larger droplets. We then generated droplets at a fixed vibrating frequency of $50 \mathrm{~Hz}$ and measured the droplet sizes at different flow rates from 1 to $500 \mathrm{~nL} / \mathrm{s}$. The linear correlation between the droplet volume and flow rate matched very well with theoretical values calculated by eq 1 (Figure 3c). Therefore, the droplet size is predictable and controllable by $\mathrm{XiE}$, which remarkably simplifies the droplet volume control.

Factors Influencing the Droplet Monodispersity. We next tested several factors that might influence the monodispersity of droplets generated by XiE. First, we generated droplets in oils of different measured viscosities (5.5, 11, 20, 44.2 , and $70 \mathrm{mPa} \cdot \mathrm{s}$ at $25^{\circ} \mathrm{C}$ ), which were prepared by mixing mineral oils with $n$-tetradecane at different ratios. As shown in Figure $4 \mathrm{a}-\mathrm{f}$, at a flow rate of $50 \mathrm{~nL} / \mathrm{s}$ and vibrating frequency of $50 \mathrm{~Hz}$, droplets generated in different oils were of the same size with diameters of approximately $124 \mu \mathrm{m}$, and the CVs from all experiments were similar $(\sim 2 \%)$. This suggested that the oil viscosity within the tested range did not affect the droplet volume or monodispersity. However, oils with a relatively low viscosity and density are preferred in the $\mathrm{XiE}$ system, which allowed quick settlement of droplets to the bottom of the microwell.

We next investigated the impact of aqueous phase viscosity on droplet formation. Three aqueous phases were used: water, $20 \%(\mathrm{v} / \mathrm{v})$, and $50 \%(\mathrm{v} / \mathrm{v})$ glycerol solutions in water with calculated viscosities of $0.89,1.73$, and $6.88 \mathrm{mPa} \cdot \mathrm{s}$ (at $25^{\circ} \mathrm{C}$ ), respectively. ${ }^{23}$ At a flow rate of $10 \mathrm{~nL} / \mathrm{s}$ and vibrating frequency of $50 \mathrm{~Hz}$, droplets generated using individual aqueous phases were measured. All droplets were of similar sizes with an average diameter of $70 \mu \mathrm{m}$, and the CV was between 2 and 3\%, indicating that the droplets were uniform (Figure $4 \mathrm{~g}-\mathrm{i}$ ). These results suggested that the viscosity of the aqueous phase did not alter the droplet size or monodispersity. Our results also 

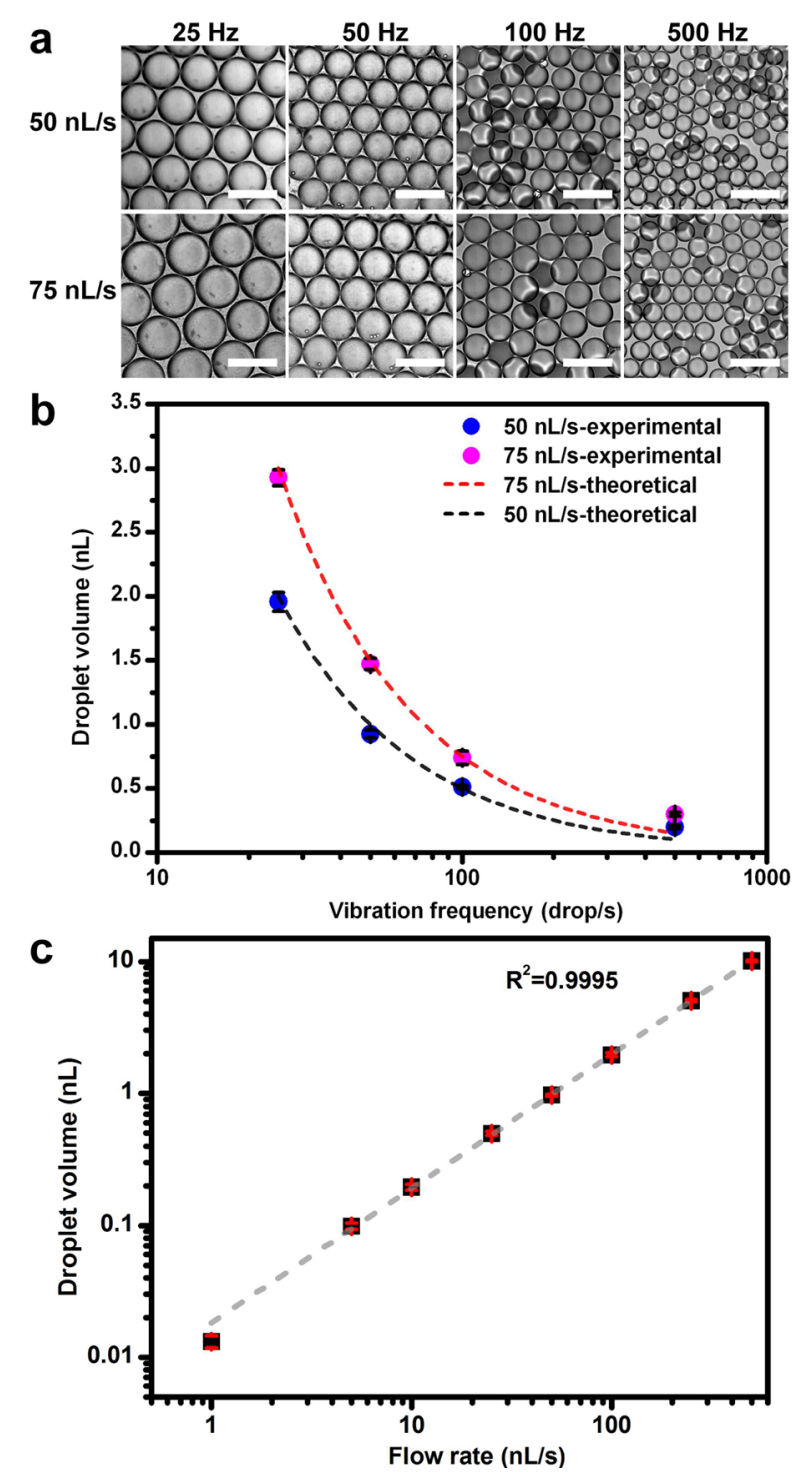

Figure 3. Droplet size is determined by the flow rate and vibrating frequency. (a) Micrographs of monodisperse droplets generated by $\mathrm{XiE}$ under different vibration frequencies and flow rates. Scale bar $=$ $200 \mu \mathrm{m}$. (b) The correlation among the droplet volume, flow rate, and vibrating frequency. Dashed lines represent theoretical calculations. (c) The linear correlation between the droplet volume and flow rate at a fixed vibrating frequency of $50 \mathrm{~Hz}$. The dashed line represents the theoretical calculation.

suggested that the $\mathrm{XiE}$ system might be applicable to viscous biological fluids, such as blood.

We next tested if the tip geometries of the capillary have significant influences on the size of the droplets. First, we prepared two capillaries with the same $40 \mu \mathrm{m}$ i.d. but different outer diameters, including a capillary with a tapered $57 \mu \mathrm{m}$ o.d. and a capillary with a flat $127 \mu \mathrm{m}$ o.d. (Figure 5a). Droplets were generated with two capillaries by the $\mathrm{XiE}$ system at different flow rates; the droplet sizes were measured, and volumes were calculated, which were compared with theoretical values derived from eq 1 . Droplets produced by the two capillaries did not show an obvious difference in size at flow rates between 5 and $100 \mathrm{~nL} / \mathrm{s}$ (Figure $5 \mathrm{~b}$ ). However, the flat capillary showed better consistency when the flow rates were higher than $100 \mathrm{~nL} / \mathrm{s}$, as it produced droplets with sizes closer to the theoretical values. Likewise, the tapered tip out- performed the flat one when the flow rate was smaller than 5 $\mathrm{nL} / \mathrm{s}$. Furthermore, we tested a series of tapered capillaries with different inner diameters, including 20, 40, 60, and $100 \mu \mathrm{m}$ (Figure 5c), and they all showed similar performance when the flow rates were between 10 and $100 \mathrm{~nL} / \mathrm{s}$. A capillary tip with a $100 \mu \mathrm{m}$ i.d. was too large for producing picoliter droplets $(20$ and $100 \mathrm{pL}$ ), which affected the droplet monodispersity when flow rates were smaller than $10 \mathrm{~nL} / \mathrm{s}$; larger inner diameters were more suitable when the droplet volumes were higher than $5 \mathrm{~nL}$. Droplets generated at $10 \mathrm{~nL} / \mathrm{s}$ were $20 \mathrm{pL}$ according to eq 1 and our experiments, and even smaller droplets could also be obtained by further reducing the flow rate. Therefore, $\mathrm{XiE}$ was competent to produce droplets with volumes over a large range, from tens of picoliters to tens of nanoliters, by simply adjusting the flow rate.

Overall, our results demonstrated that, within the tested conditions, the droplet monodispersity is independent of the oil viscosity and the properties of the aqueous solution and only weakly affected by the capillary diameters, reflecting the stability and capacity of XiE.

Self-Assembly of the PMDA. Self-assembly of the PMDA is critical for imaging and statistical analysis of droplets, and it is a distinct feature of $\mathrm{XiE}$ (Figure 1c). Because the density of oil was lower than the aqueous solution, the droplets spontaneously descended to the flat-bottom of the microwell (6600 $\mu \mathrm{m}$ in diameter) and gradually spread out and self-assembled into a tightly packed planar droplet array (Figure 1c). To determine the maximum number $(N)$ of equal-sized droplets that could be completely encompassed by the microwell of the plate without overlapping, we utilized the best-known solutions for "circle packing in a circle problem" from Eckard Specht's Web site (http://hydra.nat.uni-magdeburg.de/packing/cci/cci. html) (see Text S2). Data from the web site were used to plot the relation between the droplet number $(N)$ for a PMDA and the ratio $(\mathrm{Ra})$ of the diameter of the microwell over the droplet (Figure S2a). We then performed experiments to investigate the actual relation between the injection volume and the flow rate with regard to forming the PMDA. The target volumes over, equal to, and below the theoretical value were tested for flow rates of $25,50,70,95,200,300$, and $500 \mathrm{~nL} / \mathrm{s}$, at vibration frequency of $50 \mathrm{~Hz}$. The injection target volumes best filling the microwells without overlapping were $2.0,2.5,2.8,3.1,3.9,4.4$, and $5.3 \mu \mathrm{L}$, respectively. Other injection volumes resulted in either insufficient packing or overlapping of droplets in the microwells. The experimental results corresponded well with the theoretical predictions (Figure S2b). Overall, by setting parameters of the flow rate and corresponding injection target volume, droplets with volumes from 1 to $10 \mathrm{~nL}$ could automatically form PMDAs in flat-bottomed microwells.

We observed that the formation of the PMDA was related to the size of the droplets. Large droplets formed PMDAs rapidly; smaller droplets with volumes less than $1 \mathrm{~nL}$ required more time to spread. As shown in Movie S4, droplets of $1 \mathrm{~nL}$ volume formed PMDAs within $10 \mathrm{~min}$. When droplet volume decreased to $0.4 \mathrm{~nL}$, the droplets was not fully spread out on the bottom of the microwell after $30 \mathrm{~min}$, which failed to form a PMDA. Heating, shaking, or using oils with lower viscosity may promote the formation of PMDAs.

Multivolume dLAMP. We performed dLAMP for the absolute quantification of DNA templates using XiE. Serial dilutions of the lambda DNA stock solution, ranging from $60 \mathrm{fg}$ to $60 \mathrm{pg}$, were used as templates in the dLAMP experiments. Seven premixed LAMP reactions, including the no-template 

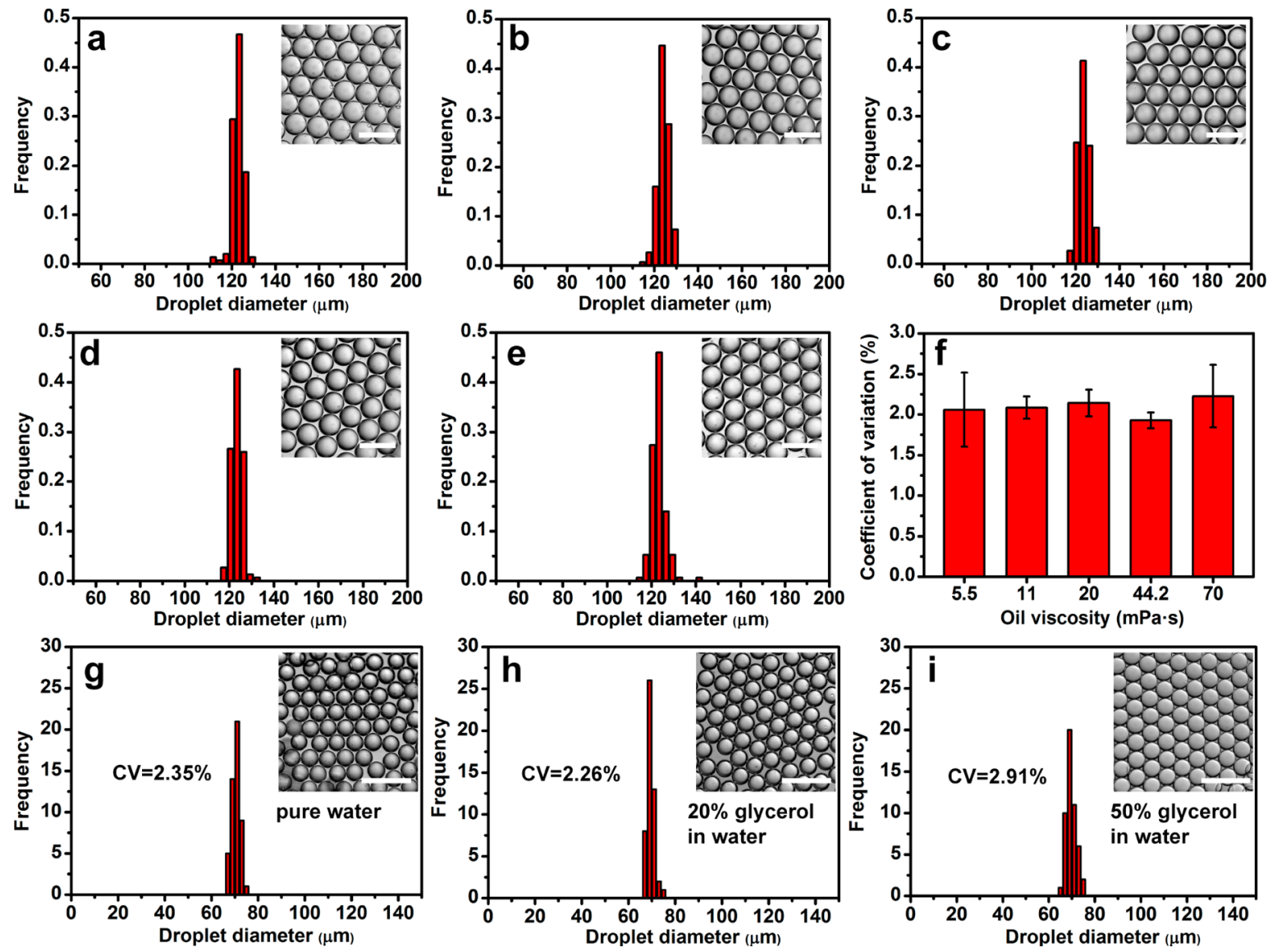

Figure 4. Consistency of the diameters of droplets generated by XiE using oil phases with different viscosities of (a) 5.5, (b) 11, (c) 20, (d) 44.2, and (e) $70 \mathrm{mPa} \cdot \mathrm{s}$ at a flow rate of $50 \mathrm{~nL} / \mathrm{s}$ and $50 \mathrm{~Hz}$ vibration. (f) Comparison of the coefficient of variation (CV) of droplet diameters derived from experiments $(\mathrm{a}-\mathrm{e})$. The droplet size and monodispersity is consistent among aqueous phases of different viscosities: $(\mathrm{g}) 0.89 \mathrm{mPa} \cdot \mathrm{s}(\mathrm{water}),(\mathrm{h})$ $1.73 \mathrm{mPa} \cdot \mathrm{s}$ (20\% glycerol in water), and (c) $6.88 \mathrm{mPa} \cdot \mathrm{s}$ ( $50 \%$ glycerol in water) at a flow rate of $10 \mathrm{~nL} / \mathrm{s}$ and $50 \mathrm{~Hz}$ vibration. Scale bars for the inserted images in $(\mathrm{a}-\mathrm{e})$ and $(\mathrm{g}-\mathrm{i})=200 \mu \mathrm{m}$.

control, were encapsulated into monodisperse droplets by $\mathrm{XiE}$ individually, forming PMDAs on a 96-well plate prefilled with the oil. The plate was incubated at $65{ }^{\circ} \mathrm{C}$ for $60 \mathrm{~min}$, and no droplet fusion was observed under this condition using the surfactants in oil. The oil coverage also prevented droplet evaporation and cross-contamination. The subdivision of reactions in nanoliter droplets resulted in some of the droplets containing a single copy of the template DNA, which were illuminated by fluorescence after amplification, whereas others with no DNA template showed no fluorescence. Signals were collected using a fluorescence microscope and analyzed by ImageJ to determine the number of positive as well as the total droplet number (Figure 6a). Fluorescence signals were evident and displayed an approximate 3 -fold change between positive and negative droplets (Figure S3). In the droplet-based digital quantification system, the concentration of the template molecule is calculated according to Poisson distribution. ${ }^{24}$ The measured template concentration was plotted against the input amount, obtaining a linear fit curve $\left(R^{2}=0.9915\right)$ (Figure $6 \mathrm{~b})$. When droplets were $1 \mathrm{~nL}$ in volume, digital quantification was successfully performed for the input DNA of less than 60 pg. PDMAs with smaller droplets $(0.5 \mathrm{~nL})$ were required and displayed better resolution for the digital quantification of the $60 \mathrm{pg}$ input DNA because the positive ratio almost reached saturation when using $1 \mathrm{~nL}$ droplets and yielded an inaccurate concentration of 3149 copies $/ \mu \mathrm{L}$, whereas $0.5 \mathrm{~nL}$ droplets yielded 2955 copies $/ \mu \mathrm{L}$, which showed a better linear agreement with other tested concentrations.

The consistency and accuracy of the multivolume dLAMP were also confirmed by the quantification of input lambda DNA of $0.3 \mathrm{pg} / \mu \mathrm{L}$ using droplets of $0.2,0.5,1$, and $2 \mathrm{~nL}$. All volumes displayed excellent digital resolution and yielded almost the same concentration of approximately 154 copies $/ \mu \mathrm{L}$ with a coefficient of variation of $0.89 \%$ (Figure S4). The absolute quantification dynamic range could be further scaled up by decreasing the droplet volume and therefore increasing the total droplet amount of the PMDA. Therefore, by simply controlling the size and quantity of droplets, this $\mathrm{XiE}$ system can provide multivolume PMDAs for the absolute quantification of DNA templates with high resolution and adaptive dynamic range.

\section{CONCLUSIONS}

We have developed the $\mathrm{XiE}$ method for the rapid generation of monodisperse droplets with unique features of on-demand control of the volume and quantity of droplets to be generated in one experiment. The proper control of droplet size and number allows self-assembly of PMDAs in flat-bottomed reservoirs. Using $\mathrm{XiE}$, we successfully performed digital loopmediated isothermal amplification (dLAMP) for the absolute quantification of nucleic acids. The system is simple, stable, 
a

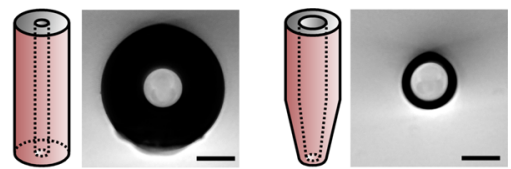

b
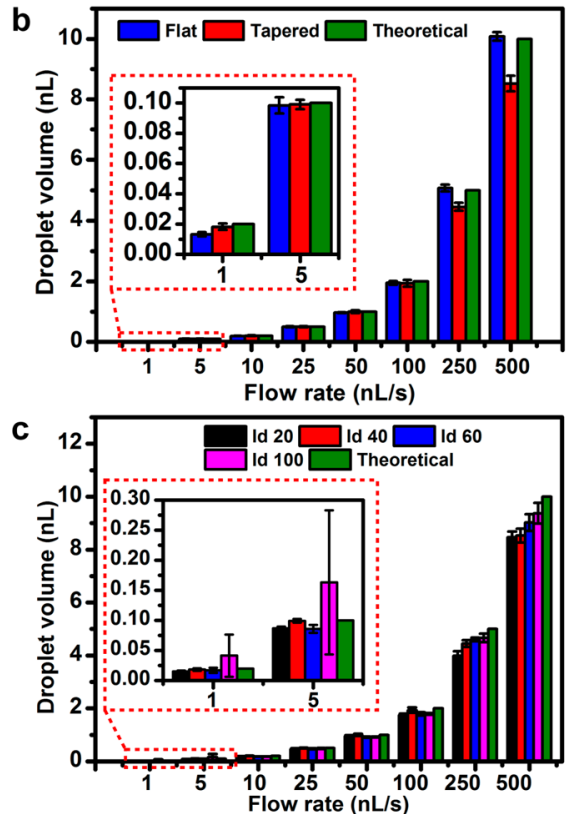

Figure 5. Droplet formation is insensitive to inner and outer diameters of the capillary tip. (a) Schematics and microscopic images of the flat and tapered capillaries with the same i.d. of $40 \mu \mathrm{m}$ but different outer diameters. Scale bar $=50 \mu \mathrm{m}$. (b) Droplets were generated using capillaries in (a). (c) Droplets were generated using tapered capillaries with different inner diameters and flow rates. Dotted rectangles in (b) and (c) showing an expanded view of the columns at flow rates smaller than $10 \mathrm{~nL} / \mathrm{s}$.

flexible, and inexpensive. Therefore, it can be readily extended to a wide range of applications.

The size of the droplets is determined principally by the flow rate and vibrating frequency, and can be easily tuned by adjusting these two parameters, which is different from other microfluidic methods. Using devices with higher vibration frequencies and vibrating a bundle of capillaries at the same time instead of one may increase the throughput of the droplet generation and offer more options for the control of droplet sizes. $\mathrm{XiE}$ is competent to produce droplets with volumes in a wide range, from picoliters to nanoliters. Extra-fine tips with both small i.d. and o.d. may help generate even smaller droplets, and therefore enlarge the dynamic range of bioassays by $\mathrm{XiE}$. In this work, the emulsification by $\mathrm{XiE}$ produced waterin-oil (W/O) droplets. Similarly, oil-in-water (O/W) droplets can also be obtained by flowing an oil phase through the capillary tip during its vibration across the air/water interface with surfactants added to the water.

A number of attempts have been made for performing digital PCR $^{19,25-28}$ or isothermal amplifications ${ }^{29-35}$ for the absolute quantification of nucleic acids using microfluidic techniques. Several commercial platforms are available for performing droplet-based digital PCR. For example, the Bio-Rad QX200 system generates $20,0001 \mathrm{~nL}$ droplets in $20 \mu \mathrm{L}$ reactions, and it takes $\sim 2$ min for each set of eight samples $(\sim 45 \mathrm{~min}$ for a full 96-well plate). Meanwhile, multivolume digital assays were introduced on SlipChip devices for the quantification of nucleic acids with wider dynamic ranges. ${ }^{36,37}$ In comparison, XiE offers
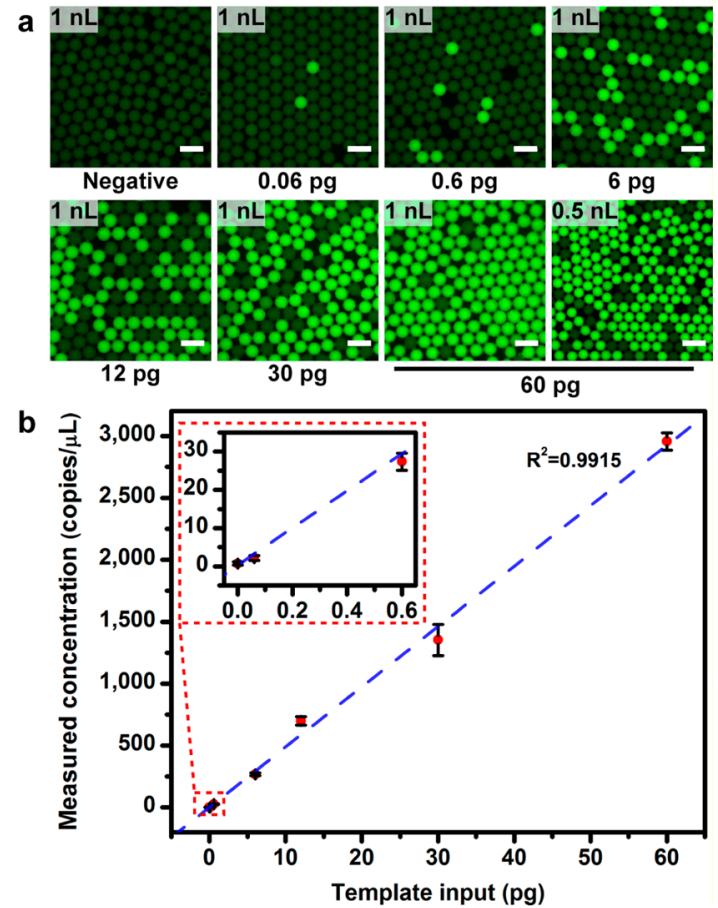

Figure 6. Digital loop-mediated isothermal amplification (dLAMP) based on PMDAs generated by XiE. (a) Fluorescence images of the PMDAs after the dLAMP reactions. Scale bar $=200 \mu \mathrm{m}$. (b) The linear correlation (blue dashed line) between the input and the measured concentration of lambda DNA templates based on dLAMP. Dotted red rectangle showed an expanded view of the correlation at low template concentration.

a simple and flexible strategy to generate size-tunable droplet arrays independent of device design for multivolume digital analysis and may promote the general application of digital nucleic acid quantification with adaptive dynamic ranges. To generate 20,000 droplets in one sample, it takes approximately $40 \mathrm{~s}$ at $500 \mathrm{~Hz}$ vibration. Compared with the time scale of PCR reactions, which usually take $1-3 \mathrm{~h}$, the time for $\mathrm{XiE}$ should be acceptable by most users. A remaining challenge is to generate droplets of multiple samples $(8,24$, or even 96$)$ simultaneously on 96-well plates using multichannel designs as well as a robotic system for automated sample loading.

We performed multivolume dLAMP as a representative application of the $\mathrm{XiE}$ method for the absolute quantification of nucleic acids in biological samples. Although LAMP has many advantages, contamination control is still challenging. ${ }^{38} \mathrm{XiE}$ offers excellent contamination control because either the capillary outlet or the droplets are covered by the oil phase at all times, which was demonstrated by our experiment results. Additionally, droplet generated by $\mathrm{XiE}$ may be transferred to other droplet-manipulating devices for functional operations, such as reading, mixing, and sorting, which might lead to more sophisticated applications, such as rapid detection and analysis of pathogens, gene sequencing, immunoassays, and single cell analysis.

\section{ASSOCIATED CONTENT}

\section{S Supporting Information}

The Supporting Information is available free of charge on the ACS Publications website at DOI: 10.1021/acs.analchem.5b04510. 
Investigation of physical phenomena of droplet segmentation and self-assembly of the PMDA in a flat-bottomed 96-well plate (PDF)

Bright-field photographs taken at 59 frames per second showing droplet generation in a microwell prefilled with oil (AVI)

Bright-field microscopic images taken at 4,800 frames per second showing details of droplet segmentation at the capillary tip under the surface of oil (AVI)

Bright-field microscopic images taken at 6,000 frames per second showing confinement of droplets in the oil phase when the capillary is lifting (AVI)

Bright-field microscopic images of PMDAs with two different droplet volumes, taken at $1 \mathrm{~min}$ intervals, showing that PMDA formation is dependent on the droplet size (AVI)

\section{AUTHOR INFORMATION}

\section{Corresponding Author}

*E-mail: wenbin@im.ac.cn. Telephone: +86-10-82994175.

Notes

The authors declare no competing financial interest.

\section{ACKNOWLEDGMENTS}

This work was supported by the program of China Ocean Mineral Resources R\&D Association (DY125-15-R-02), the Strategic Priority Research Program of Chinese Academy of Sciences (XDB15040102), the National Natural Science Foundation of China (21205134, 31470221, 11202219, and 11572335), the National High Technology Research and Development Program of China (2012AA092103), and the CAS Interdisciplinary Innovation Team.

\section{REFERENCES}

(1) Bibette, J.; Calderon, F. L.; Poulin, P. Rep. Prog. Phys. 1999, 62, 969-1033.

(2) Tadros, T. F. Emulsion science and technology; Wiley-VCH: Weinheim, 2009.

(3) Bai, S.; Pappas, C.; Debnath, S.; Frederix, P. W.; Leckie, J.; Fleming, S.; Ulijn, R. V. ACS Nano 2014, 8, 7005-7013.

(4) Harrison, R. G.; Washburn, A. L.; Pickett, A. T.; Call, D. M. J. Mater. Chem. 2008, 18, 3718-3722.

(5) El Kinawy, O. S.; Petersen, S.; Helmdach, L.; Ulrich, J. Chem. Eng. Technol. 2012, 35, 1604-1608.

(6) Shanmugam, A.; Ashokkumar, M. Food Hydrocolloids 2014, 39, 151-162.

(7) Maindarkar, S. N.; Bongers, P.; Henson, M. A. Chem. Eng. Sci. 2013, 89, 102-114.

(8) Higashi, S.; Shimizu, M.; Nakashima, T.; Iwata, K.; Uchiyama, F.; Tateno, S.; Tamura, S.; Setoguchi, T. Cancer 1995, 75, 1245-1254.

(9) Chen, D.; Pui, D. Y. H.; Kaufman, S. L. J. Aerosol Sci. 1995, 26, 963-977.

(10) Sun, Y.; Chen, X.; Zhou, X.; Zhu, J.; Yu, Y. Lab Chip 2015, 15, 2429-2436.

(11) Teh, S.; Lin, R.; Hung, L.; Lee, A. P. Lab Chip 2008, 8, 198220.

(12) Thorsen, T.; Roberts, R. W.; Arnold, F. H.; Quake, S. R. Phys.

Rev. Lett. 2001, 86, 4163-4166.

(13) Anna, S. L.; Bontoux, N.; Stone, H. A. Appl. Phys. Lett. 2003, 82, 364-366.

(14) Cramer, C.; Fischer, P.; Windhab, E. J. Chem. Eng. Sci. 2004, 59, 3045-3058.

(15) Squires, T. M.; Quake, S. R. Rev. Mod. Phys. 2005, 77, 9771026
(16) Utada, A. S.; Lorenceau, E.; Link, D. R.; Kaplan, P. D.; Stone, H. A.; Weitz, D. A. Science 2005, 308, 537-541.

(17) Gu, H.; Duits, M. H.; Mugele, F. Int. J. Mol. Sci. 2011, 12, 25722597.

(18) Chiu, D. T.; Hsiao, A.; Gaggar, A.; Garza-Lopez, R. A.; Orwar, O.; Zare, R. N. Anal. Chem. 1997, 69, 1801-1807.

(19) Hatch, A. C.; Fisher, J. S.; Tovar, A. R.; Hsieh, A. T.; Lin, R.; Pentoney, S. L.; Yang, D. L.; Lee, A. P. Lab Chip 2011, 11, 38383845.

(20) Waldbauer, G. A walk around the pond:insects in and over the water; Harvard University Press: Cambridge, MA, 2006.

(21) Garstecki, P.; Fuerstman, M. J.; Stone, H. A.; Whitesides, G. M. Lab Chip 2006, 6, 437-446.

(22) Tabeling, P. Phys. Fluids 2010, 22, 021302.

(23) Cheng, N. Ind. Eng. Chem. Res. 2008, 47, 3285-3288.

(24) Bhat, S.; Herrmann, J.; Armishaw, P.; Corbisier, P.; Emslie, K. R. Anal. Bioanal. Chem. 2009, 394, 457-467.

(25) White, R. R.; Blainey, P. C.; Fan, H. C.; Quake, S. R. BMC Genomics 2009, 10, 116.

(26) Shen, F.; Du, W.; Kreutz, J. E.; Fok, A.; Ismagilov, R. F. Lab Chip 2010, 10, 2666-2672.

(27) Men, Y.; Fu, Y.; Chen, Z.; Sims, P. A.; Greenleaf, W. J.; Huang, Y. Anal. Chem. 2012, 84, 4262-4266.

(28) Baker, M. Nat. Methods 2012, 9, 541-544.

(29) Sun, B.; Shen, F.; McCalla, S. E.; Kreutz, J. E.; Karymov, M. A.; Ismagilov, R. F. Anal. Chem. 2013, 85, 1540-1546.

(30) Rane, T. D.; Chen, L.; Zec, H. C.; Wang, T. H. Lab Chip 2015, $15,776-782$.

(31) Zhu, Q.; Gao, Y.; Yu, B.; Ren, H.; Qiu, L.; Han, S.; Jin, W.; Jin, Q.; Mu, Y. Lab Chip 2012, 12, 4755-4763.

(32) Khorosheva, E. M.; Karymov, M. A.; Selck, D. A.; Ismagilov, R. F. Nucleic Acids Res. 2016, 44, e10.

(33) Shen, F.; Davydova, E. K.; Du, W.; Kreutz, J. E.; Piepenburg, O.; Ismagilov, R. F. Anal. Chem. 2011, 83, 3533-3540.

(34) Jarvius, J.; Melin, J.; Goransson, J.; Stenberg, J.; Fredriksson, S.; Gonzalez-Rey, C.; Bertilsson, S.; Nilsson, M. Nat. Methods 2006, 3, 725-727.

(35) Gansen, A.; Herrick, A. M.; Dimov, I. K.; Lee, L. P.; Chiu, D. T. Lab Chip 2012, 12, 2247-2254.

(36) Kreutz, J. E.; Munson, T.; Huynh, T.; Shen, F.; Du, W.; Ismagilov, R. F. Anal. Chem. 2011, 83, 8158-8168.

(37) Shen, F.; Sun, B.; Kreutz, J. E.; Davydova, E. K.; Du, W.; Reddy, P. L.; Joseph, L. J.; Ismagilov, R. F. J. Am. Chem. Soc. 2011, 133, 17705-17712.

(38) Tomita, N.; Mori, Y.; Kanda, H.; Notomi, T. Nat. Protoc. 2008, $3,877-882$ 